\title{
Is the EPR paradox a paradox?
}

\author{
A. Tartaglia, Dip. Fisica, Politecnico, Corso Duca degli Abruzzi 24, I-10129 Torin
}

January 1, 2018

\begin{abstract}
The EPR paradox and the meaning of the Bell inequality are discussed. It is shown that considering the quantum objects as carrying with them "instruction kits" telling them what to do when meeting a measurement apparatus any paradox disappears. In this view the quantum state is characterized by the prescribed behaviour rather than by the specific value a parameter assumes as a result of an interaction.
\end{abstract}

\section{Introduction}

A famous paper by Einstein, Podolsky and Rosen [1] appeared in 1935 in the Physical Review gave rise to a live debate on the meaning and the foundation of quantum mechanics, lasting up to our days. Einstein, Podolsky and Rosen (EPR) pointed out that something must be missing in the quantum theory because applying it to a thought experiment they analysed, led to a paradox or at least to a contradiction with the Heisenberg uncertainty principle. Three decades later, J. Bell proved his celebrated inequality [2] and applying it to the EPR experiment concluded that in some cases quantum mechanics violates it and that its violation would imply a break down either of the principle of reality or of the principle of locality (provided one would not be inclined to give up causality). Different variants of the EPR experiment have been considered such as the one proposed by Bohm [3] using pairs of spin $1 / 2$ particles prepared in a singlet state. Finally A. Aspect and his group, in the 80's, actually performed a series of EPR type experiments measuring the polarization of pairs of photons produced in an SPS atomic cascade [4]. They verified that the result of the experiments was correctly predicted by quantum theory and that in some configurations Bell's inequality was actually violated. 
All this strengthened the idea that quantum mechanics is indeed paradoxical in character and revived a debate whose ingredients are mainly the supposed nonlocality of quantum phenomena, if not the existence of some sort of superluminal interaction between distant quantum objects.

Now what is rather surprising in this story is that the so called EPR paradox appears as being mainly due to a misuse of the Heisenberg principle which should have been seen since the very beginning. A vague idea of this may be found in the answer by Bohr [5] to EPR's paper.

The claim that the paradox was not there and simply was a consequence of an incorrect application of the rules of probability was first issued by L. Accardi [6] who developed an axiomatic approach to what he calls quantum probability [7].

In the present paper I shall show why the EPR paradox is not a paradox and why the result of Aspect's experiments does not imply any violation of the principle of locality. In the next section the basic properties of a quantum object or system are reviewed. In the third section the EPR type experiments are analysed, then section 4 contains a discussion of the meaning of the Bell inequality; finally section 5 summarizes the whole paper.

\section{Quantum objects}

A quantum object is something that can exist in a set of different physical states. Mathematically these states can be eigenstates of a number of different operators. Practically the effect of any quantum operator corresponds to the interaction with a given apparatus which induces the object to enter one of the eigenstates of the corresponding operator.

We know that operators exist which are mutually incompatible in a sense that they do not admit common eigenstates. The commutator of two such operators differs from zero: if $\widehat{A}$ and $\widehat{B}$ are the two operators, applying them in the order $\widehat{A}$ $\widehat{B}$ leaves the system in a state different from the one in which it is left applying them in the order $\widehat{B} \widehat{A}$.

All this means that when the system is in an eigenstate of $\widehat{A}$, the physical quantity associated to that operator assumes one of its eigenvalues, say $a$, but the quantity associated with $\widehat{B}$ is undefined. Conversely, when the system is in an eigenstate of $\widehat{B}$, the quantity it supports assumes the eigenvalue $b$, but now the quantity associated with $\widehat{A}$ is undefined.

This is of course the Heisenberg principle. It is appropriate to stress that there is nothing mysterious, exotic or peculiar to quantum physics in this. It has 
simply been stated, in a refined and mathematically precise way, the fact that anybody may be standing or seated, but nobody may simultaneously be standing and seated.

\section{The EPR paradox}

In their thought experiment EPR consider a couple of particles moving in opposite directions and so prepared that their total momentum is zero. If we measure the momentum of one of them we know the momentum of the other without measuring it. Now if we determine the position of the second particle, we end up knowing both position and momentum of one and the same object, thus contradicting the Heisenberg principle.

Actually performed experiments use rather pairs of objects prepared in a singlet state or photons emitted in an SPS atomic transition, so that their spins are antiparallel (first case) or polarization vectors equal (second case). Once the two objects are well separated one from the other each of them is tested, using an appropriate analyser, for the spin component or polarization state along a given direction. Now using the EPR argument, if the measurements are performed along different directions (for instance orthogonal directions) we conclude that the spin components or polarization states of one object along different directions can be simultaneously determined, in one case by direct measurement and in the other by a counterfactual but (apparently) sound inference.

The week point in this is the idea of "element of reality" (to use EPR's words) whose value is being tested or inferred. Einstein (and many others after him) think of the experimentally testable properties of a quantum system as being things carried around by the system: they are objectively there, measure them or not. Now this idea appears to be in contrast with experience, because of the "paradoxes" it generates.

Actually the situation is different. We may think that a quantum object (which is well defined and existent, independently from the fact of being under measurement or not) carries its properties as an "instructions kit" telling it what to do when encountering a measuring apparatus or in general undergoing an interaction with something else.

In Aspect's experiments the two photons, at the act of separating, receive, each of them, their "instructions" (whose content depends on the way they are prepared). When they meet an analyser they behave accordingly: the realism is save and Einstein's locality is save too. If the two objects meet two experimental 
sets of the same kind so are the results (in case of spin components, they may have the same or opposite sign, depending on the initial preparation). If however one of the members of the pair is led to interact with a different type of apparatus, its "instruction card" tells it to behave differently.

Now the counterfactual inference that, in the EPR argument, lead to the "paradox" tells us what the object under attention would have done if it had been subjected to an experiment different from the one actually performed; nothing more then this. Our quantum object possesses nothing else than its unique and not contradictory "instructions kit": no two incompatible behaviours can be adopted in the same time. The Heisenberg principle is save.

This way of reading things dissolves the EPR paradox and has no particular subtlety in it; furthermore it is neither particularly exotic nor confined to the quantum domain.

To express ideas of the same kind as those presented in this paper Accardi uses a [simpatica] metaphor: that of the chameleons [8]. A chameleon is a well defined non quantum "object" which has the property of assuming the colour of the support on which it rests. Now suppose you have a pair of twin chameleons and separate them far away one from the other, then put one of them on a leaf: it will become green. We can conclude, without experiment, that the second chameleon, if it had been put on a leaf, it would have become green too, not certainly that it is green. Actually suppose somebody puts the second chameleon on a trunk: it will become brown. Nobody, knowing the result of both experiments, would conclude that the second chameleon is green and brown at the same time. The point is that the chameleon does not possess a colour, but the capacity to assume a colour according to the environment in which it is laid. Everybody will agree: 1) that the chameleons are perfectly real and existing at any moment; 2) that the phenomenon we verified is purely local; 3 ) that there is no logical contradiction to be accounted for.

There is no reason that what is true for chameleons (or other classical and macroscopic systems) cannot be true for electrons or photons. On the contrary there are good reasons it to be true for them also.

\section{Bell inequality}

The Bell inequality has been considered as being a cornerstone in the understanding of the nature and properties of quantum objects and phenomena. This concept has been rather emphatically expressed in the statement by Stapp that Bell's is 
"the most profound discovery of science" 9].

Bell originally applied his analysis to the EPR experiment. Maybe the clearest way to expound it is to consider the Aspect's version of that experiment. The core of the argument moves from the results of three sets of measurements, two being actually performed and the third being inferred on the basis of the original preparation of the pair of objects being tested. The procedure is rather idealized and the physical quantities and features of the experiment are so devised that the issues of each of the three actual or hypothetical measurements (let us call them $a, b$ and $c$ ) may only be \pm 1 . Everything then rests on the identity 10:

$$
a(b-c) \equiv \pm(1-b c)
$$

Repeating the experiment many times, then considering statistical averages of the results one arrives at

$$
|\langle a b\rangle-\langle a c\rangle| \leq 1-\langle b c\rangle
$$

which is the Bell inequality.

Using quantum mechanics to evaluate the averages one sees both by calculation (as Bell himself did) and by experiment (Aspect and coworkers) that in some situations (4.2) is violated.

Now, according to Bell and to many people after him, the only hypotheses underlying identity (4.1) are:
a) realism
b) determinism
c) locality

Since (4.1) proves to be false and (4.2) violated, at least one of the hypotheses, it is said, must be false too. The one usually doomed to be abandoned is the third, i.e. locality (often called Einstein locality). This introduces action at a distance and consequently violates the principle of relativity forcing people to formulate various kinds of arguments intended to demonstrate that the action at a distance is actually there but cannot really be used to transmit informations at a speed higher than that of light.

Actually however there is a fourth hidden (to say better: not declared) hypothesis underlying Bell's reasoning: it is the assumption that the property being 
measured is something possessed as such by the quantum object and being there at any moment. This means that, even if, say, $a$ and $c$, on one side, $b$ and $c$, on the other, cannot be measured simultaneously, provided a rule exists enabling us to guess what the result had been if $c$ had been measured instead of a (or $c$ instead of $b$ ), we are entitled to use both $a$ and $c$ (or $b$ and $c$ ) at the same time in (4.1).

The situation is different if we think quantum objects as carrying, so to speak, recipe books rather than cakes, as said in the previous section. A recipe produces a cake only when the right kitchen and ingredients are met and it has no meaning to correlate a cake (= result of a measurement) with a recipe different from the one being used. Out of metaphor, identity (4.1) is now deprived of sense and so is the ensuing inequality (4.2). The logic of recipe books is different from the one of cakes.

There is nothing strange in this, nor peculiar to the quantum world, as Accardi's chameleons show, and all three conditions (4.3) are fully satisfied.

\section{Conclusion}

Since the very beginning quantum mechanics subverted deeply rooted ways of thinking and mental [abitudini]. This involved its phenomena and theory in a halo of mystery, giving rise to a decades long debate and a host of different interpretations of quantum phenomena calling into play free will, the consciousness of the experimenter and things like that. Now that the shock of the subversion of the bald programs of the XIX century physics is far behind us, a quiet reconsidering of some of the "strange" behaviours of quantum objects shows that they are neither really that strange nor confined to the quantum world. An example is the famous EPR paradox which is indeed no paradox, provided we accept that the state of a quantum objects is defined by what I called an "instruction kit" or a "recipe book". In other words, the spin or the polarization are a behaviour while interacting rather than a colour or a label attached to a particle.

Looking at things from this viewpoint, we see that realism, determinism and localism are generally satisfied conditions (as we reasonably expect) and that some features of the quantum phenomena may again find their counterpart in the macroscopic domain.

This does not mean that everything is clear and solved with quantum mechanics: the meaning of the wave function, its collapse, the quantization of the objects it describes still escape our imagination and, at least in my case, our understanding. Nonetheless we may claim that part of the mystery is only apparent and 
comes from a sort of long lasting prejudice.

In conclusion quantum measurements and the story of the violation of the Bell or similar inequalities tell us that the objects of the quantum world are not like boxes containing spin, polarization vector etc. like buttons, pins, pearls and the like, but like programmed machines capable of different behaviours according to the physical conditions locally triggering them. To mock the beautiful Accardi's metaphor I would say that the quantum particles are "camleons" rather than "boxons".

\section{References}

[1] Einstein A, Podolsky B and Rosen N 1935 Phys. Rev. 47777

[2] Bell J S 1964 Physics 1195

[3] Bohm D 1951 Quantum Theory (New York: Prentice Hall)

[4] Aspect A, Grangier P and Roger G 1982 Phys. Rev. Lett., 49 91;

Aspect A, Dalibard G and Roger G 1982 Phys. Rev. Lett. 491804

[5] Bohr N 1935 Phys. Rev. 48696

[6] Accardi L 1984 The probabilistic roots of the quantum mechanical paradoxes in The wave-particle dualism ed S Diner et al (Reidel Publishing Company) p. $297-330$

[7] Accardi L 1982 Foundations of quantum probability in Rend. Sem. Mat. Univ. Polit. Torino (Torino: Levrotto \& Bella) p. 249-270

[8] Accardi L 1997 Urne e camaleonti (Milano: Il Saggiatore)

[9] Stapp H P 1975 Nuovo Cimento B 29270

[10] Peres A 1993 Quantum Theory: Concepts and Methods (Dordrecht: Kluwer Academic Publishers) p. 